\title{
Coarse Integral Imaging without Pseudo Image
}

\author{
Tomoya Kurokawa* and Hideki Kakeya \\ University of Tsukuba \\ 1-1-1 Tennoudai, Tsukuba 305-8573, JAPAN
}

\begin{abstract}
Coarse integral imaging (CII), where each elemental lens is large enough to cover pixels far more than the number of views, can show clear floating 3D image when distortion is corrected. One of the major problems left to be solved for $\mathrm{CII}$ is suppression of pseudo images that appear around the right image to be presented. In this paper we propose two methods to suppress pseudo images. We first propose use of a lens array with a small $\mathrm{F}$ number. When a lens array composed of elemental lenses whose $\mathrm{F}$ number is small is set in front of the display panel, pseudo images can be erased by total internal reflection on the outskirt of the large aperture lens because the angle of incidence of the light ray that generates pseudo images becomes larger. The second method we propose is use of a lens array behind the display panel paired with segmented backlight. When convex lenses are set in front of the backlight with limited aperture, leak of ray out to adjacent elemental lenses can be avoided. Since the backlight area is reduced, this method can also reduce consumption of electric power without diminishing brightness of the right image.
\end{abstract}

Keywords: coarse integral imaging, coarse integral volumetric imaging, optics, real image, pseudo image, total internal reflection, lens array, resolution

\section{INTRODUCTION}

Integral imaging [1], which combines fly-eye lenses with a high resolution flat display panel, is a prominent 3D display system in the sense that it can show not only horizontal parallax but also vertical parallax. In the conventional integral imaging, the number of pixels each elemental lens of the fly-eye lens sheet covers is usually the same as the number of views, which means that the viewer perceives each elemental lens as one pixel. Therefore the focus of the viewer's eyes is always fixed on the screen (fly-eye lens sheet), which makes it hard to show realistic images far beyond the screen or popping up from the screen.

Besides the orthodox integral imaging described above, we can also think of integral imaging where each elemental lens is large enough to cover pixels dozens of times more than the number of views, which has been studied by Lee et al. [2-4]. Kakeya defined this type of integral imaging as coarse integral imaging (CII) [5]. The advantage of CII is that it can induce focal accommodation off the screen, for it generates a real image or a virtual image with the lenses. Thus we can show realistic images far beyond the screen or popping up from the screen.

Coarse integral volumetric imaging (CIVI) combines volumetric solution with the multiview solution based on CII $[5,6,7]$. To realize CIVI, layered transparent display panels are used instead of a single layer display panel in CII. When we use multi-layered display panels, we can show volumetric real image or virtual image. CIVI can reduce the contradiction between binocular convergence and focal accommodation that arises in multiview 3D displays, and can express occlusion or gloss of the objects in the scene, which can not be expressed with volumetric 3D displays. Thus we can overcome the shortcoming of multiview displays and volumetric displays at the same time.

One major problem left to be solved for CII and CIVI is suppression of the pseudo images that appear around the right image to be presented. The right image of CII and CIVI is generated when the light ray from the elemental image goes through the elemental lens right above it, while the pseudo images emerge when the elemental image goes through the elemental lenses surrounding the right elemental lens. Therefore pseudo image can be suppressed if the barrier is set between the elemental images and the elemental lenses so that the ray from each elemental image may not leak out to adjacent elemental lenses. This solution, however, is hard to realize practically because the barrier has to have complex physical structure. In the present paper, we propose two methods to suppress emergence of pseudo images. The first method applies total internal reflection of lenses and the second method uses a lens array paired with backlight with limited aperture behind the display panel.

*E-mail : s1020855@u.tsukuba.ac.jp Phone\&FAX :+81-29-853-5255 
This paper is organized as follows. In section 2 the principle of CII and CIVI is reviewed. In section 3 we propose and explain suppression of pseudo images by applying total internal reflection of the lenses. In section 4 we propose and explain suppression of pseudo images by using a lens array paired with limited backlight. In section 5 summary of this paper is given.

\section{COARSE INTEGRAL IMAGING}

\subsection{Principle of CII}

Coarse integral imaging (CII) is a type of integral imaging where coarse fly-eye lens sheet (convex lens array) is used instead of a fine fly-eye lens sheet, which is used for conventional integral imaging. In the conventional integral imaging, the number of pixels each elemental lens of the fly-eye lens sheet covers is usually the same as the number of views. The viewer perceives each elemental lens as one pixel and the focus of the viewer's eyes is always fixed on the screen. In CII, however, each elemental lens covers dozens by dozens or hundreds by hundreds of pixels. The observable number of pixels through each elemental lens is equal to the number of pixels covered by each lens divided by the square of magnifying power of the optical system. For example, when 100X100 pixels are covered by each lens of the fly-eye lens sheet and the magnifying power given by the pair of lenses (elemental lens and large aperture lens) is 10, 10X10 pixels are observed through each elemental lens.

With CII we can express 3D space far beyond the screen or popping up from the screen. To present 3D space far beyond the screen we can use virtual image, which is generated when the distance between the display panel and the lens sheet is nearer than the focal length of the elemental lenses of the lens sheet. We can express 3D objects popping up from the screen by generating real image, which emerges when the distance between the display panel and the lens sheet is farther than the focal length of the elemental lenses of the lens sheet.

In the actual generation of real image for CII, we usually use a large aperture Fresnel lens in addition to the fly-eye lens sheet to converge light, for the aberration becomes small with this configuration. The distance between the display panel and the lens array is kept almost the same as the focal distance of the elemental lenses of the lens array so that the light may be collimated by each elemental lens. Then the large aperture convex Fresnel lens converges the collimated light and generates a real image at the focal distance of the Fresnel lens away from its surface.

The distance between the large aperture convex lens and the fly-eye lens sheet can vary. When the distance is short, the optics is similar to that of the traditional integral imaging and the total system size can be kept compact, while it can be a multiview system where the whole image observed in each eye switches alternately when we keep the distance between the lens array and the large aperture Fresnel lens long enough to generate the real image of the lens array, which corresponds to the viewing zone where the view for each eye changes alternately.

Though CII can show images off the screen, the problem of vergence-accommodation conflict still exists, for it can only generate one image plane. Besides the vergence-accommodation conflict, CII has another major problem that can severely damage the quality of the image. When the distance between the lens array and the large aperture Fresnel lens is not far enough, multiple images from different elemental lenses are observed at the same time. In this case discontinuity of the images from different lenses becomes severe when the 3D image to be shown has large depth. To show depth of the image, CII depends only on the parallax included in multiview images. The parallax among the images from different lenses has to be larger as the depth of the 3D object to be shown becomes longer. Consequently discontinuity of the images on the boundaries of the lenses ruins the quality of image.

\subsection{Principle of CIVI}

To solve the problem of vergence-accommodation conflict and discontinuity of image at the same time, Kakeya et al. proposed coarse integral volumetric imaging (CIVI), which is based on the idea of introducing volumetric approach in addition to the multiview approach [6,7]. As shown in Figure 1 multiple display panels are inserted to generate volumetric real image (volumetric virtual image can also be generated if the display panels and the lens array are set closer) to keep the parallax between the images from two adjacent lenses small enough. Since artificial parallax is kept small in this system, discontinuity between images from adjacent lenses can be reduced. Vergence-accommodation conflict is also reduced since each 3D pixel is displayed at the real image (virtual image) layer near the right depth. 
To express pixels between two panels we can use DFD approach $[8,9,10]$, where 3D pixels are expressed with two adjacent panels, each of which has the pixel intesity in inverse proportion to the distance between the 3D pixels and the panel. Thus natural continuity of depth is realized.

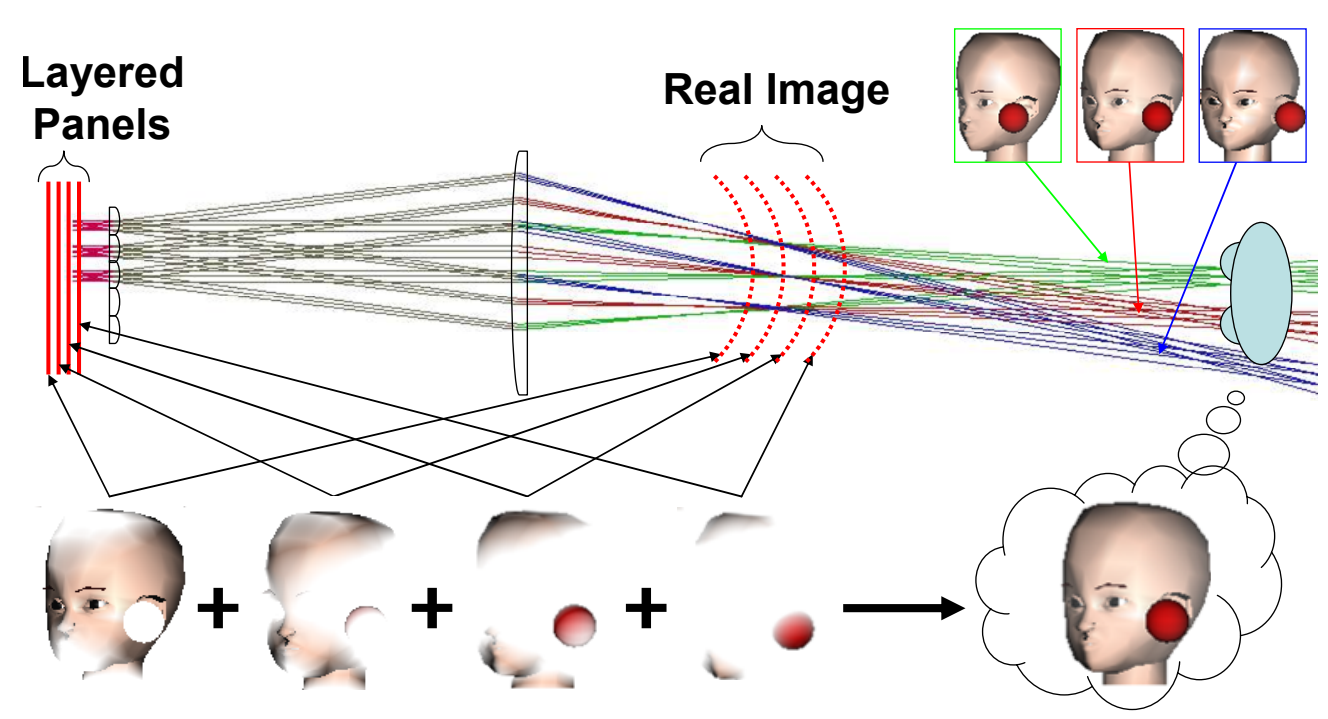

Figure 1. Principle of coarse integral volumetric imaging.

The major problem remaining for CII and CIVI is emergence of pseudo images that appear around the right image to be presented as shown in Figure 2 (A). The right image of CII and CIVI is generated when the elemental image goes through the elemental lens right above it, while pseudo images emerge when the elemental image goes through the elemental lenses surrounding the right elemental lens as shown in Figure 2 (B). In the following sections we discuss how to suppress these pseudo images.

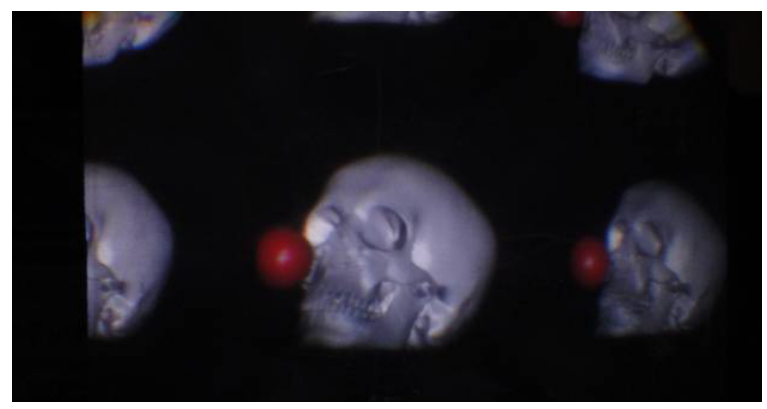

(A)

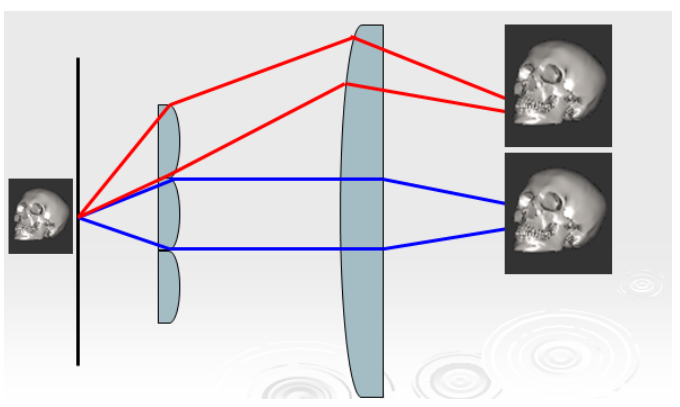

(B)

Figure 2. (A) Pseudo images observed in CII and CIVI displays and (B) Mechanism of how pseudo images emerge.

\section{SUPRESSION OF PSEUDO IMAGES BY TOTAL INTERNAL REFLECTION}

\subsection{Principle}

The first method we propose to suppress pseudo images is related to total internal reflection. When a lens array composed of elemental lenses whose $\mathrm{F}$ number is small is set in front of the display panel, pseudo images can be erased by total internal reflection on the outskirt of the large aperture lens because the angle of incidence of the light ray that 
generates pseudo images becomes larger. When F number is not small enough to cause total internal reflection, we can still make the pseudo images less distinct because pseudo images are generated far behind the large aperture lens as long as F number is relatively small compared with the conventional CII/CIVI systems.

Figure 3 illustrates how pseudo images become less distinct by using elemental lenses with small $\mathrm{F}$ numbers. As shown in Figure 1, real images of the display panels generated by the lenses are curved. This curvature becomes steeper as the focal length of the lens becomes shorter. Then the pseudo images go beyond the surface of the large aperture lens and are located on the surface that faces in the direction almost perpendicular to the viewer. Thus pseudo images become almost invisible from the viewer. If the focal length becomes still shorter, pseudo images disappears completely due to the total internal reflection.

When elemental lenses with small $\mathrm{F}$ numbers are used, distortion of image becomes severe not only in the direction of depth, but also within the image plane [11]. This distortion can be corrected by using texture mapping technique $[12,13]$.
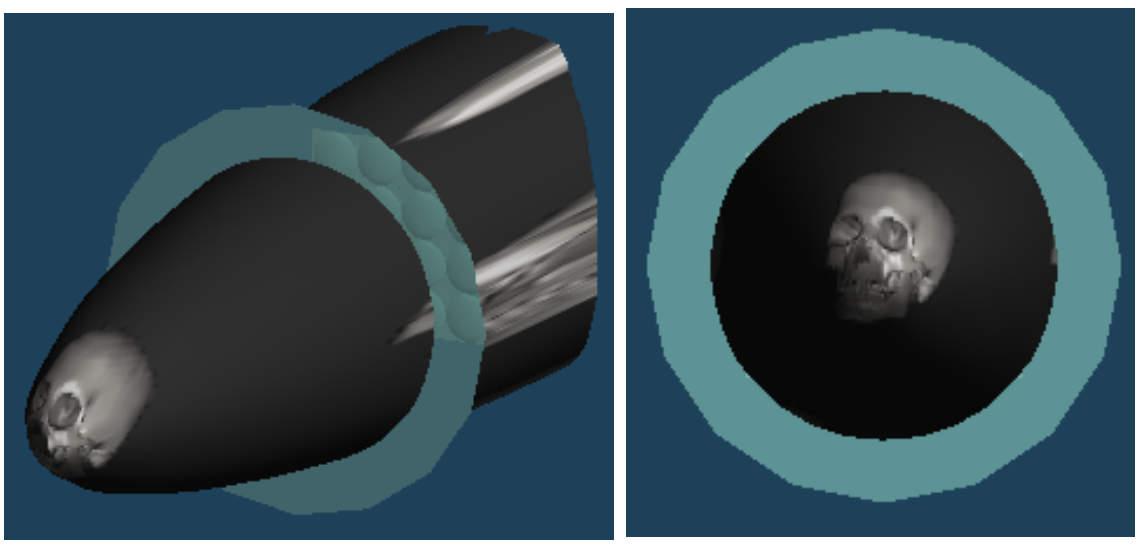

Figure 3. Pseudo images generated by lenses with short focal distances. The ring in the above picture shows the surface of the large aperture lens.

\subsection{Prototypes}

In this study we made three prototypes using elemental lenses with different $\mathrm{F}$ numbers, and observed the right images and pseudo images generated by them. In all the prototypes, 8.4 inch TFT color LCD monitors (TOTOKU PLT210-B0X, resolution: $1024 \times 768$ ) are used as the display panel and the focal distance of the large aperture lens is fixed to $325 \mathrm{~mm}$. Focal parameters of the lenses used for the prototype systems and the conventional system [11] are listed in Table 1.

When $\mathrm{F}$ number of elemental lenses is small, pseudo images are expected to become less distinct. On the other hand, the images generated by lenses with short focal distances have strong distortion. Therefore these two factors should be balanced to realize high quality image and suppression of pseudo image at the same time.

Table 1. Lens parameters.

\begin{tabular}{|l|l|l|l|}
\hline & Focal distance (mm) & Lens aperture (mm) & F number \\
\hline Prototype A & 25 & 22 & 1.14 \\
\hline Prototype B & 50 & 30 & 1.67 \\
\hline Prototype C & 60 & 32 & 1.875 \\
\hline Conventional system [11] & 160 & 36 & 4.44 \\
\hline
\end{tabular}


In the first prototype (prototype A), F number of the elemental lens is set to 1.14 . This prototype has the smallest F number of the three. Figure 4 shows the pictures of the prototype hardware and the observed images with this prototype. Although pseudo images are erased, the right image presented by this prototype has only poor resolution. In the CII and CIVI displays, the size of the image observed from each viewpoint is the same as the size of each elemental image multiplied by the magnifying power of the lenses. Since the distance between the elemental lens and the elemental image is the same as the focal distance of the elemental lens, the magnifying power $\mathrm{M}$ is given by

$$
\mathrm{M}=\frac{F_{a}}{F_{b}},
$$

where $F_{a}$ is the focal distance of large aperture lens and $F_{b}$ is the focal distance of the elemental lenses. In Prototype A, the image is magnified by the lens system by 13 times $(325 \mathrm{~mm} / 25 \mathrm{~mm})$, which means that dot pitch is also magnified by 13 times, which makes the pixel dot distinct.
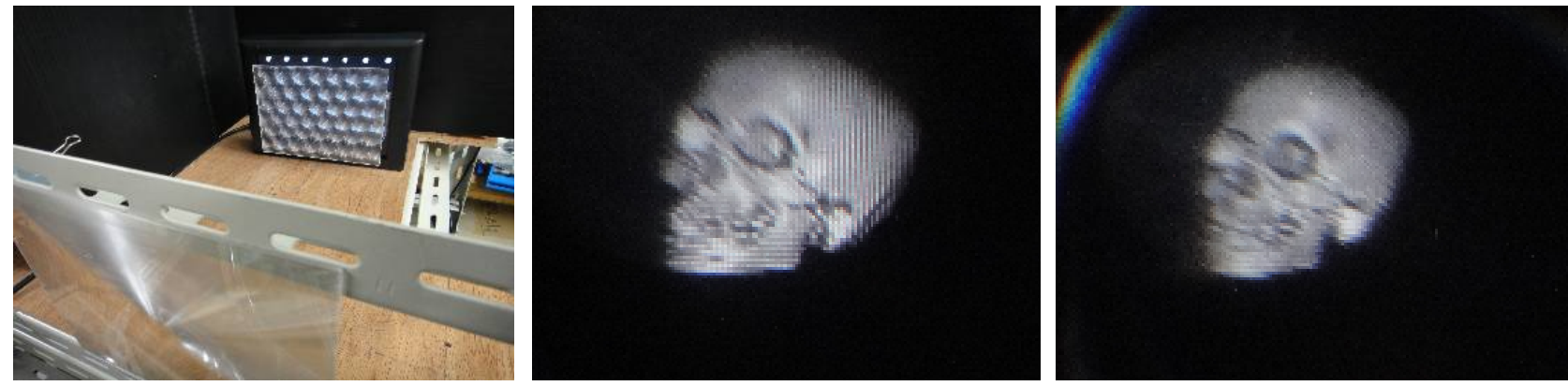

Figure 4. Prototype system A (left) and the images observed from central viewpoint (middle)

and a far-left viewpoint (right) with this system.

Prototype B has an F number between prototypes A and C. Figure 5 shows the images observed with this prototype system. Compared with prototype A, we can see finer image because of its smaller magnifying power, while pseudo images appears instead. However, pseudo images do not stand out and are hard to be detected by the observer.
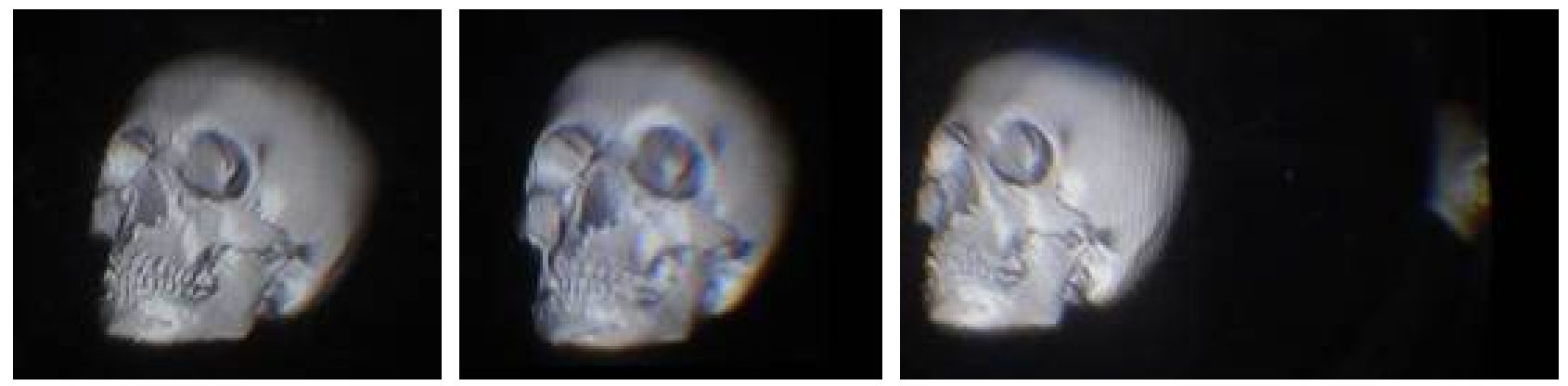

Figure 5. The images of prototype B observed from the central viewpoint (left), a far-left viewpoint (middle) and the viewpoint including both the right image and a pseudo image (right).

Prototype $\mathrm{C}$ has the largest $\mathrm{F}$ number of the three. Figure 6 shows the images observed with this prototype. We can see clear images whose resolution is the highest because of the least magnification of pixel dots of the three prototypes. On the other hand, pseudo images become the most distinct. Even though pseudo images become more distinct they still do not stand out and do not annoy the viewer. 

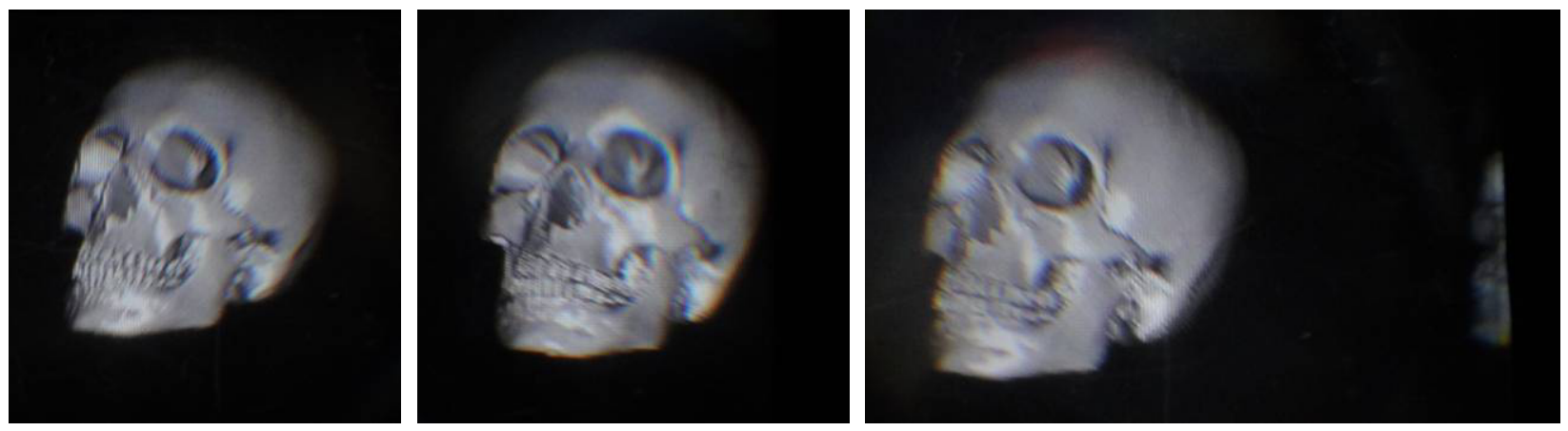

Figure 6. The images of prototype $\mathrm{C}$ observed from the central viewpoint (left), a far-left viewpoint (middle) and the viewpoint including both the right image and a pseudo image (right).

With the prototypes described above, it is confirmed that the method we propose can suppress pseudo images. However, the trade off between suppression of pseudo image and the resolution of the presented right image have to be balanced in this system. Though prototypes $\mathrm{B}$ and $\mathrm{C}$ can show clear image, the resolution of the image is inferior to that of the conventional CIVI displays because of its greater magnifying power. In Section 4, the authors propose another method to suppress pseudo images, which is free from the problem of lower resolution.

\section{SUPRESSION OF PSEUDO IMAGE BY LIMITED BACKLIGHT}

\subsection{Principle}

Though the method proposed in Section 3 can suppress pseudo images easily just by using elemental lenses with small $\mathrm{F}$ numbers, the resolution of the image becomes poorer. In this section we propose another method to suppress pseudo images, which can secure the same resolution as the conventional CII and CIVI systems. The method we propose is illustrated in Figure 7. As this figure shows, another lens array is set behind the display panel showing the elemental images so that each lens of the lens array covers each elemental image. Behind the additional lens array is set a backlight with limited aperture. With this configuration the light rays that go through each elemental image can reach only the corresponding elemental lens and do not reach nearby elemental lenses. Thus it is expected that light rays generating pseudo images are suppressed and those generating right images are maintained.

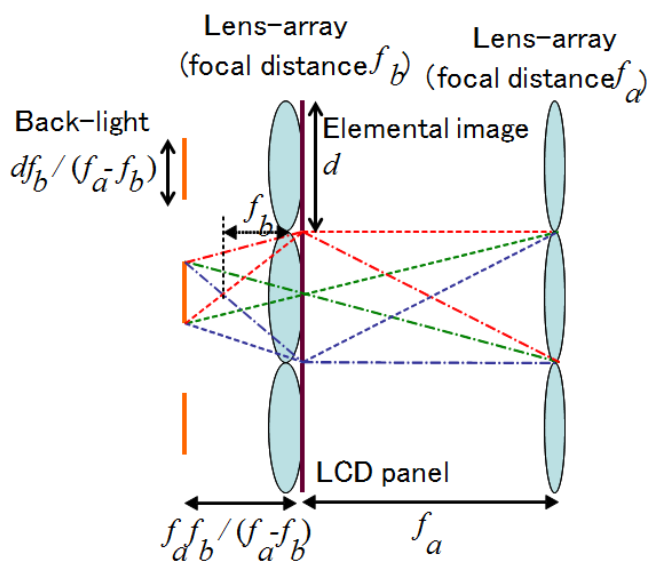

Figure 7. Principle of pseudo image suppression by use of lens array coupled with limited backlight. 
Here we explain in detail how the limited backlight paired with the lens array can attain suppression of pseudo images. Let the focal distance and the diameter of elemental lenses be $f_{a}$ and $d$ respectively. Also let us assume that the distance between the display panel showing elemental images and the lens sheet of elemental lenses is $f_{a}$. By simple discussion on geometrical optics as shown in Figure 7, leak of ray out to adjacent elemental lenses and loss of ray into proper elemental lens are both avoided when the diameter $r$ of segmented backlight is given by

$$
r=\frac{d^{*} f_{b}}{\left(f_{a}-f_{b}\right)},
$$

and the distance $l$ between the backlight and the display panel is written in the form

$$
l=\frac{f_{a} * f_{b}}{\left(f_{a}-f_{b}\right)},
$$

where $f_{b}$ is the focal distance of the lens adhered behind the display panel.

Since the backlight area is reduced, this method consumes less electric power. Here the brightness of the presented image is not reduced because what is cut is limited to the light source for pseudo images. Thus it is expected that we can realize power-efficient display system with this method.

\subsection{Prototype}

Based on the above principle, we made a prototype system. Figure 8 shows the pictures of the prototype system hardware and the observed images with this prototype. Here an 8 inch color LCD monitor (CENTUREY LCD-8000U, resolution : $800 \times 600$ ) is used as for the display panel and the focal distance of the large aperture lens is $325 \mathrm{~mm}$. As for the lens-array in front of the elemental images, lenses $30 \mathrm{~mm}$ in diameter and $100 \mathrm{~mm}$ in focal distance are used, and as for the lens-array behind the elemental images, lenses $30 \mathrm{~mm}$ in diameter and $12 \mathrm{~mm}$ in focal distance are used. For simplicity, this prototype realizes limited area of backlight by setting frames with limited aperture in front of the backlight to confirm whether pseudo image is suppressed as expected. To attain energy-efficient system we need to use an array of point light sources in place of the backlight we use for this prototype.
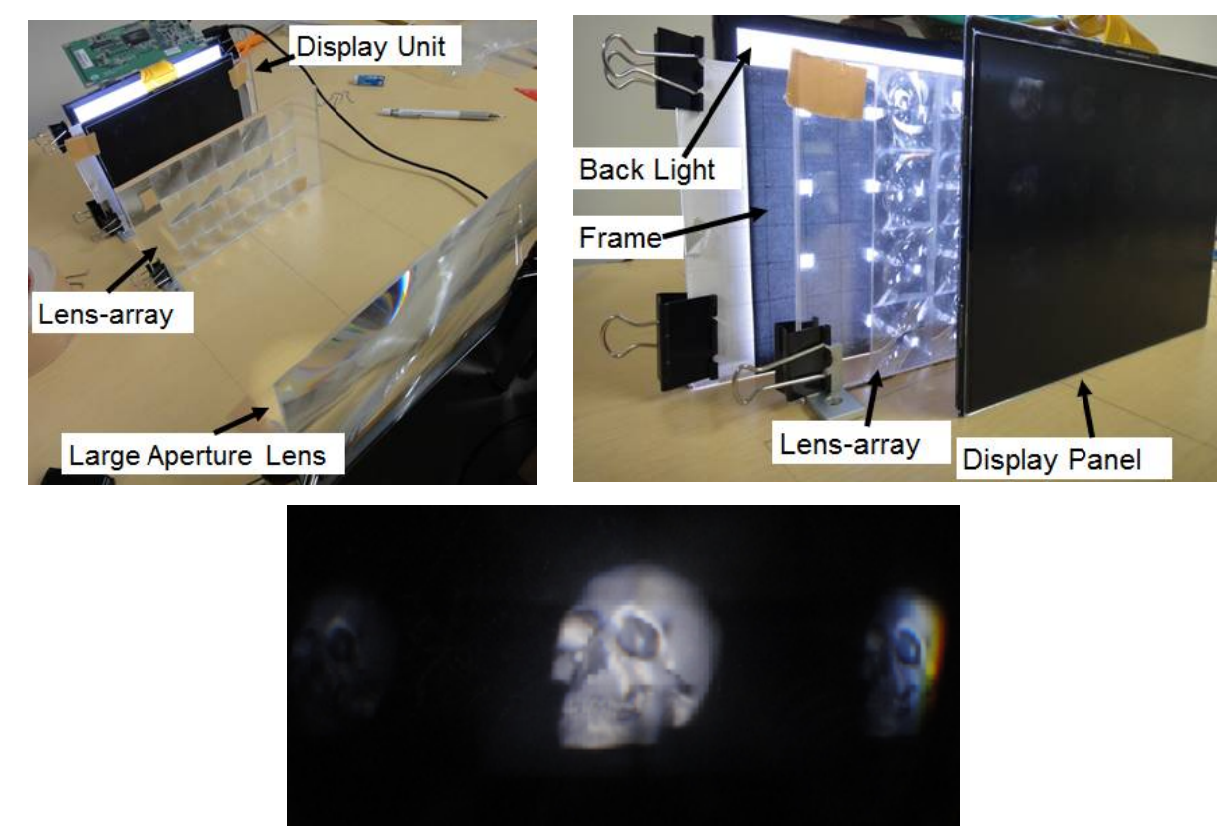

Figure 8. Pictures of the prototype hardware (top left and right) and the image observed with this prototype (bottom). 
As Figure 8 shows, pseudo image is suppressed to a certain extent, but not eliminated completely. Also we can see dark bands in the seam of the elemental lenses. We can think of two reasons for these problems. One is the inaccuracy of the placement of aperture and lenses and the other is the optical aberration. Figure 9 illustrates why pseudo images remain and dark bands emerge. In theory, the rays from segmented backlight area reach only the proper lenses. However, it is practically hard to cover just only the proper lens because of inaccuracy of placement and aberration. If the aperture of the backlight is too small and the proper lens is not covered perfectly, the dark band appears in the seam of the elemental lenses. On the other hand, if the aperture of the backlight it too large and the ray leaks out to adjacent elemental lenses, it causes emergence of pseudo images. Further study is needed to realize suppression of pseudo images without disturbing the right image practically with this method.

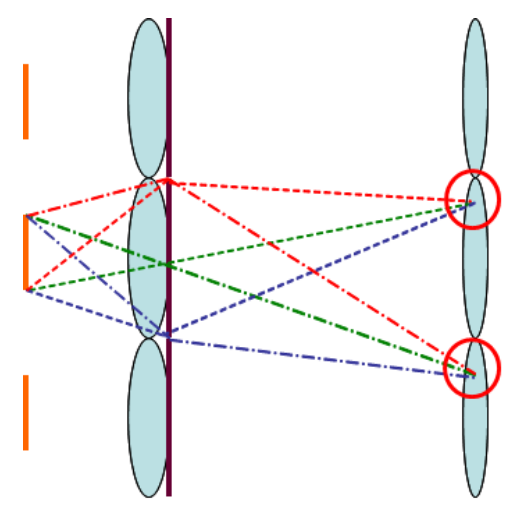

Figure 9. Illustration of how pseudo images and black bands emerge.

\section{SUMMARY}

In this present paper, we propose two methods to suppress pseudo images for CII and CIVI displays. In the first method use of a lens array with a small $\mathrm{F}$ number is considered. When a lens array composed of elemental lenses whose $\mathrm{F}$ number is small is set in front of the display panel, pseudo images disappear or become less distinct on the outskirt of the large aperture lens. It is confirmed with the three prototype systems based on this method that the pseudo images fades as expected, though the resolution becomes lower compared with the conventional CII and CIVI displays. The second method we propose uses a lens array behind the display panel paired with segmented backlight. When the lens is paired with a limited backlight, leak of ray out to adjacent elemental lenses can be avoided. With the prototype system it is confirmed that pseudo images are suppressed but not eliminated completely. Further study is needed to realize high resolution images and suppression of pseudo images at the same time.

\section{ACKNOWLEDGEMENT}

This research is partially supported by the Grant-in-Aid for Scientific Research, MEXT, Japan, Grant number: 22680008 .

\section{REFERENCES}

[1] G. Lippmann, "La photograhie integrale," Comptes Rendus Acad. Sci. 146, 446-451 (1908).

[2] B. Lee, S. Jung, S.-W. Min, and J.-H. Park, "Three-dimensional display by use of integral photography with dynamically variable image planes," Opt. Lett. 26, 1481-1482 (2001).

[3] J.-H. Park, S. Jung, H. Choi, and B. Lee, "Integral imagingwith multiple image planes using a uniaxial crystal plate," Opt. Express 11, 1862-1875 (2003). 
[4] S.-W. Min, B. Javidi, and B. Lee, "Enhanced three-dimensional integral imaging system by use of double display devices,"Appl. Opt. 42, 4186-4195 (2003).

[5] H. Kakeya, "Coarse integral imaging and its applications", SPIE proceeding Volume 6803: Stereoscopic Displays and Virtual Reality Systems XV, 680317 (2008).

[6] H. Kakeya, "Improving Image Quality of Coarse Integral Volumetric Display", SPIE proceeding Volume 7237 : Stereoscopic Displays and Virtual Reality Systems XVI, 723726 (2009).

[7] H. Kakeya, T. Kurokawa, Y. Mano, "Electronic Realization of Coarse Integral Volumetric Imaging with Wide Viewing Angle", SPIE proceeding (2010)

[8] S. Suyama, H. Takada, K. Uehira, S. Sakaiand S. Ohtsuka, "A Novel Direct-Vision 3-D Display using Luminance-Modulated Two 2-D Images Displayed at Different Depths," SID’00 Digest of Technical Papers, 54.1, pp. 1208-1211, (2000).

[9] S. Suyama, H. Takada and S. Ohtsuka, "A Direct-Vision 3-D Display Using a New Depth-fusing Perceptual Phenomenon in 2-D Displays with Different Depths," IEICE Trans. on Electron., Vol. E85-C, No. 11, pp.1911-1915 (2002).

[10] S. Suyama, S. Ohtsuka, H. Takada, K. Uehira and S. Sakai, "Apparent 3-D image perceived from luminance-modulated two 2-D images displayed at different depths," Vision Research, 44, pp. 785-793 (2004).

[11] H. Kodaira, A. Nakao, Y. Mano and H. Kakeya, "Distortion correction of coarse integral volumetric imaging with large lens", Proc. Of 3DSA, pp. 205-208 (2010).

[12] H. Kakeya and Y. Arakawa, "Autostereoscopic Display with Real-image Virtual Screen and Light Filters," SPIE Proceedings Volume 4660 (2002)..

[13] H. Kakeya, "Real-Image-Based Autostereoscopic Display Using LCD, Mirrors, and Lenses," SPIE Proceedings Volume 5006 (2003). 